\title{
Deflationism beyond arithmetic
}

\author{
Kentaro Fujimoto ${ }^{1,2}$
}

Received: 7 December 2016 / Accepted: 8 July 2017 / Published online: 17 August 2017 (C) The Author(s) 2017. This article is an open access publication

\begin{abstract}
The conservativeness argument poses a dilemma to deflationism about truth, according to which a deflationist theory of truth must be conservative but no adequate theory of truth is conservative. The debate on the conservativeness argument has so far been framed in a specific formal setting, where theories of truth are formulated over arithmetical base theories. I will argue that the appropriate formal setting for evaluating the conservativeness argument is provided not by theories of truth over arithmetic but by those over subject matters 'richer' than arithmetic, such as set theory. The move to this new formal setting provides deflationists with better defence and brings a broader perspective to the debate.
\end{abstract}

Keywords Truth · Deflationism - The conservativeness argument · Axiomatic theories of truth

\section{Introduction: the conservativeness argument}

The term 'deflationism' is used to stand for many different views on truth by different philosophers. Perhaps they have only a few points in common, but many deflationists would probably agree on these two points:

The author is most grateful to Volker Halbach and Leon Horsten for invaluable and fruitful discussions and comments on the content of the present paper, and he would also like to wholeheartedly thank the two anonymous referees for their helpful and insightful comments and suggestions. He is also indebted to Catrin Campbell-Moore, Anthony Everette, and Richard Pettigrew for their helpful comments on the earlier versions of the present paper.

$\bowtie \quad$ Kentaro Fujimoto

kentaro.fujimoto@bristol.ac.uk

1 Department of Philosophy, University of Bristol, Cotham House, Bristol BS6 6JL, UK

2 School of Mathematics, University of Bristol, University Walk, Bristol BS8 1TW, UK 
(D1) Truth is not a substantial property.

(D2) Truth owes its raison d'etre to its logico-linguistic function.

The thesis (D1) is the core doctrine of deflationism about truth. What exactly it means is not clear and may vary among deflationists, but only one implication that (D1) is alleged to have will be important in this article, namely, that we should not be able to obtain any new substantial knowledge of non-semantic facts by invoking the notion of truth. The thesis (D2) will be closely examined in Sect. 3.

Here I follow Horsten (2011) and call the function of truth logico-linguistic. Some deflationists like Field (1994, 1999) simply call it logical, but I agree with Horsten that truth is also a linguistic notion, since truth operates on linguistic entities (broadly interpreted) - the bearers of truth. Furthermore, as Halbach (2001) points out, truth can't be purely logical in the sense that it is ontologically neutral, since quite modest truth axioms imply the existence of at least two distinct objects. In this article, I assume, to avoid unnecessary complications, that the bearers of truth are sentence types; but my arguments can be applied to theories of truth with other truth bearers mutatis mutandis.

Horsten (1995), Shapiro (1998) and Ketland (1999) independently presented the socalled conservativeness argument against deflationism about truth. Their arguments are actually based upon a specific formal conception of 'theory of truth', in which theories of truth are given as a result of adding a truth predicate and its axioms on top of some axiomatic formal theory. That is to say, we first fix some recursively axiomatised theory B, such as Peano Arithmetic (PA) and Zermelo-Fraenkel Set Theory (ZF), of the subject matter in question, which is called a base theory. Then we add a truth predicate $T$ to the language $\mathcal{L}_{\mathrm{B}}$ of $\mathrm{B}$ and a recursive set $\mathcal{T}$ of axioms for $T$ to $\mathrm{B}$; we call the resulting theory an axiomatic theory of truth over $\mathrm{B}$ (or, over the subject matter in question, such as arithmetic and set theory, when we need not specify the base theory). Now, with this formal conception of 'theory of truth', the conservativeness argument aims to pose the following dilemma:

(C1) A deflationary theory $\mathrm{S}$ of truth must be conservative over its base theory $\mathrm{B}$ : that is, an $\mathcal{L}_{\mathrm{B}}$-sentence $\sigma$ must be provable in $\mathrm{B}$ whenever it is provable in $\mathrm{S}$.

(C2) However, no adequate theory of truth over $B$ can be conservative over $B{ }^{1}$

Throughout this article I take the standpoint that Azzouni (1999) calls first-order deflationist: namely, I commit myself to the ordinary effective notion of first-order logical consequence, and the word 'provable' always means the provability in the sense of the ordinary first-order logic unless otherwise specified. ${ }^{2}$

\footnotetext{
1 The conclusions that Shapiro, Horsten, and Ketland draw from this dilemma are different: Shapiro (1998) concludes that deflationism needs a strong and non-effective notion of logical consequence; Horsten (2011) regards the conservativeness argument as reductio ad absurdum of (C1); Ketland (1999) simply denies deflationism.

2 The target of the conservativeness argument is those first-order deflationists who adopt the specific axiomatic conception of 'theory of truth' described in Sects. 1-2, and I will focus on this type of theory of truth in this article. Many other types of theories of truth fall outside of the scope of the conservativeness argument. For instance, so-called 'semantic' theories of truth are concerned with providing an interpretation of the truth predicate $T$ on a given fixed model-theoretic structure of a base language $\mathcal{L}_{\mathrm{B}}$. Such a modeltheoretic structure is usually assumed to possess what we may call the reduct property in the terminology of abstract model theory: that is, it completely and invariably determines which $\mathcal{L}_{\mathrm{B}}$-sentences are designated
} 
The presupposition of the clear distinction between the base part $B$ and the truth part $\mathcal{T}$ of a theory $\mathrm{S}$ of truth is necessary for the conservativeness argument; otherwise, the conservativeness requirement $(\mathrm{C} 1)$ would make no sense. Furthermore, the distinction of the truth and base parts must be given in the way that the truth predicate $T$ is fresh to $\mathcal{L}_{\mathrm{B}}$ and $\mathrm{B}$ tells us nothing about $T$. In order for $(\mathrm{C} 1)$ to be a reasonable requirement, the base theory B must formally theorise about its subject matter (to the extent that it serves one's purpose) without any help from $T$; otherwise, truth would play a substantial ('inflationary') role in theorising about the subject matter, and new axioms $\mathcal{T}$ for such a substantial factor in the theorisation of the subject matter might well yield new theorems about the subject matter. ${ }^{3}$ Accordingly, we may also assume that $B$ is a theory of non-semantic subject matter, unless we are interested in formalising the Tarskian hierarchy of truths in which truths of higher levels are applied to truths of lower levels. The most common form of axiomatic theories of truth, such as the ones found in (Halbach 2010), is consonant with the so far described formal conception of 'theory of truth'. 4

The conservativeness argument has been criticised by Azzouni (1999), Field (1999), Tennant (2002) and others; then Horsten (2011), Shapiro (2004) and Ketland (2005, 2010) gave counterarguments to these criticisms. A plethora of arguments have been exchanged back and forth between those and many others, and the conservativeness argument forms a central topic of the debate on deflationism about truth nowadays. My view is, however, that many of these debates are placed in an inappropriate setting.

\section{Footnote 2 continued}

therein and which are not, and the interpretation of any predicate not belonging to $\mathcal{L}_{\mathrm{B}}$ has no effect on the designation of $\mathcal{L}_{\mathrm{B}}$-sentences. Hence, for semantic theories of truth, the conservativeness requirement (C1) would make no sense or just be trivially satisfied. The same applies to theories of truth with a strong non-effective logic that gives a categorical characterisation of $\mathcal{L}_{\mathrm{B}}$-theorems, such as $\omega$-logic and full secondorder logic (in the case where B is arithmetical); this is exactly why Shapiro (1998) concludes from his conservativeness argument that deflationism requires a non-effective notion of logical consequence (cf. fn 1). It goes beyond the scope of this article to discuss what form an adequate theory of truth should take, but my own view is that there are some important cases for which the axiomatic approach to truth with the ordinary effective notion of logical consequence is necessary, for example, where we consider what Vänäänen (2001) calls urlogic and truth over it: urlogic is 'the most primitive formal language we use to study the process of doing mathematics' (p. 510) and 'a formalization of the act of doing mathematics' whose semantics is 'totally informal' (p. 512). Firstly, I completely agree with Väänänen's conclusion that those strong non-effective logics are not acceptable as the logic of urlogic. Secondly, the axiomatic approach is the most natural and suitable for theories of truth over urlogic, since urlogic is maximally rich in the sense that I will discuss in Sect. 5 (cf. fn 23).

3 This point provides an immediate rebuttal to Horsten's counterargument to Field (1999) in Horsten (2011),

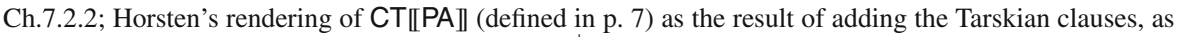
the truth axioms, on top of the base theory PA $+\mathcal{L}_{\mathbb{N}}^{+}$-Ind (defined in p. 7) violates this requirement, since this base theory already contains the truth predicate $T$, and thus the truth axioms must be added to it in terms of another truth predicate, say $T^{\prime}$.

4 There are other types of theories of truth, such as theories of Frege structures. In Frege structures, truth plays a crucially substantial role in the construction of sets: truth is used to define propositions, new sets are constructed from those propositions, truth is further applied to statements involving those newly constructed sets, from which more propositions are obtained, and this process goes on circularly or transfinitely. With this 'inflationist' conception of truth, it makes little sense to separate the non-semantic base part and the semantic truth part of these structures, and truth is not something to be added on top of a clearly separated non-semantic theory; see Aczel (1980) and Beeson (1985), Ch. XVII.7. 
The study of axiomatic theories of truth has so far centred around those over arithmetic, and philosophical debates concerning axiomatic theories of truth have been based on formal results about those theories of truth over arithmetic. The debate on the conservativeness argument so far is no exception to this trend. This is presumably not because philosophers are only interested in arithmetical truth. Rather, it is probably because they believe that theories of truth over arithmetic provide a 'generic' case and most of the relevant results concerning them and philosophical arguments based on those results can be generalised to other cases. However, I doubt the validity of this extrapolation and suspect that theories of truth over arithmetic do not constitute such a generic case. Recent research in formal logic, such as (Fujimoto 2012, 2017), reveals certain significant dissimilarities between axiomatic theories of truth over arithmetic and those over set theory, and we will see a further example of such dissimilarity in this article. These formal results indicate that theories of truth over arithmetic do not constitute such a generic case.

My proposal in this article is that the appropriate formal setting for evaluating the adequacy or inadequacy of the conservativeness argument is provided not by theories of truth over arithmetic but by those over much 'richer' subject matters such as set theory. The move to this new formal setting provides deflationists with better defence against the conservativeness argument, but the goal of this article is not to refute the conservativeness argument. My primary goal in this article is to give a closer examination of the formal assumptions upon which the conservativeness argument relies, and thereby to uncover a new area of debate on deflationism and the conservativeness argument, to which the previous debates in the literature on theories of truth over arithmetic cannot be straightfowardly generalised.

\section{The conservativeness requirement}

One peculiar but important feature of the notion of truth is its 'universality': truth is topic-neutral and can be applied to any (declarative) sentence about any subject matter. Many philosophers would probably agree on this, but we have to be careful when formally spelling it out. We have supposed that truth is a linguistic device operating on sentences. Hence, any theory of truth must be accompanied by some theory of syntax as the theory of its bearers. There are, however, different methods of incorporating a theory of syntax into a theory of truth. ${ }^{5}$ In this article, I focus on the most customary method in which the following condition (Syn) on base theories is assumed:

(Syn) A base theory contains a theory of syntax (either intrinsically or via coding), which plays the role of the theory of truth bearers, and on which the truth predicate operates.

There are two different cases to be separately considered concerning how a theory of syntax is 'contained' in a base theory. Theories of some subject matters intrinsically contain a theory of syntax per se, but others do not; for instance, a reasonably strong

$\overline{5}$ See (Achourioti et al. 2015, pp. 12-15) where three different such methods are compare and discussed. 
theory of sets intrinsically contains a theory of syntax (such as a theory of finite strings of symbols of some alphabet, where those symbols may be treated as urelements), while the intended domain of arithmetic contains nothing syntactic and a theory of natural numbers is not intrinsically concerned with syntax per se; in the latter case, a theory of syntax must be embedded in a base theory via a certain coding schema such as Gödel numbering. Note that (Syn) is a quite exclusive condition; theories of many subject matters, such as biology and medicine, do not necessarily contain any theory of syntax either intrinsically or via coding, and cannot be bases of theories of truth under the assumption of (Syn). As I will illustrate in Sect. 5, the conservativeness argument crucially relies upon the assumption of (Syn).

The universality of truth suggests that in the debate on truth we should take into account theories of truth over subject matters other than arithmetic, at least on equal terms with those over arithmetic. Even under the assumption of (Syn), we have many different theories of different subject matters available as bases of theories of truth. Hence, we have a variety of base theories that we can take in place of B in the components $(\mathrm{C} 1)$ and $(\mathrm{C} 2)$ of the conservativeness argument. However, the veracity of the claim (C2), that no adequate theory of truth over B is conservative over $B$, depends not only on what theory of truth is taken to be adequate but also on what base theory is taken in place of $\mathrm{B}$; if $\mathrm{B}$ is inconsistent, then $(\mathrm{C} 2)$ is trivially false, no matter what theory of truth is taken to be adequate; even if $B$ is consistent, we can still construct an artificial counterexample to (C2) in many cases. ${ }^{6}$ Hence, a natural question to ask is: What base theories are to be taken into account in (C1) and (C2)?

Since deflationists hold that truth is a logical device, they may well contend that it should be applicable not only to arbitrary subject matters but also to arbitrary base theories of the subject matters, as other logical devices are. Hence, a naïve answer to the aforementioned question is perhaps the following:

(C3) The requirement (C1) should be applied to any base theory B of any subject matter, as long as B meets the condition (Syn).

(C3) makes the task of the proponents of the conservativeness argument easier, since then they have only to find at least one base theory $B$ of one subject matter, from a large variety of options, such that no adequate theory of truth over B is conservative over B. I suspect that this (C3) is implicitly assumed by many proponents of the conservativeness argument and that this is why they are content to only consider a single base theory PA and take the non-conservativeness of some theories of truth over PA as a 'witness' of the substantiality of truth. One of my goals in this article is to argue that we should reject (C3) and exclude some subject matters and base theories, arithmetic in particular, from the scope of (C1).

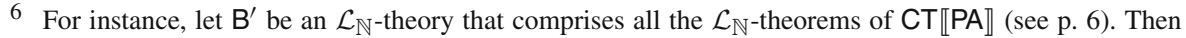
we trivially have that $C T \llbracket \mathrm{B}^{\prime} \rrbracket$ is conservative over $\mathrm{B}^{\prime}$. This theory $\mathrm{B}^{\prime}$ is arithmetically sound and primitive recursively axiomatisable; in fact, $\mathrm{B}^{\prime}$ is identical with $\mathrm{PA}$ plus the schema of transfinite induction up to $\varepsilon_{\varepsilon_{0}}$ (only for $\mathcal{L}_{\mathbb{N}}$-formulae, of course).
} 


\section{Logico-linguistic functions of truth}

In this section, I will specify one minimal requirement for adequate theories of truth through consideration of the logico-linguistic function of truth.

Deflationism about truth claims that truth is a mere logico-linguistic device. The first question to ask is: what is the logico-linguistic function of truth? It is often claimed by deflationists that truth is a device of 'indirect endorsement' and 'infinite conjunction'. The use of truth as a device of indirect endorsement is exemplified in statements like 'what Karl said at the trial is true', in which one endorses what Karl said at the trial without bothering to write down it or even without knowing exactly what he said. This function of truth is normally achieved by means of the truth predicate $T$ and definite descriptions of the sentences one wants to endorse; for instance, given a definite description $K x$ of the sentence that Karl asserted at the trial, we can formally express 'what Karl said at the trial is true' by $\forall x(K x \rightarrow T x)$. The use of truth as a device of infinite conjunction is exemplified in sentences like 'all axioms of ZF are true', in which the truth of infinitely many sentences are asserted; we first pick a predicate $\mathcal{A} x$ characterising the set of the axioms of ZF, such that $\mathcal{A} x$ holds if and only if $x$ is (a code of) an axiom of ZF, and thereby formally express the sentence by $\forall x(\mathcal{A} x \rightarrow T x)$.

The second question to ask is: what truth axioms are needed to properly implement the logico-linguistic function of truth? In this article, I will focus on two kinds of theories of truth, which are particularly at issue in the debate on the conservativeness argument. Given a base theory $\mathrm{B}$ and its language $\mathcal{L}_{\mathrm{B}}$, let $\mathcal{L}_{\mathrm{B}}^{+}$be the language of theories of truth over $\mathrm{B}$, which is obtained by adding a truth predicate $T$ or a satisfaction predicate $S a t$ to $\mathcal{L}_{\mathrm{B}}$; the choice between $T$ and $S a t$ depends on the theory of truth at stake and its specific formulation, but I will be deliberately sloppy about the distinction between them to avoid unnecessary technical complications and always assume that the truth predicate $T$ is explicitly defined in terms of Sat when Sat is taken as a primitive predicate symbol of $\mathcal{L}_{\mathrm{B}}^{+} \cdot{ }^{7}$ We first consider purely disquotational theories $\mathrm{TB} \uparrow$ and TB of truth. The theory $T B \uparrow$ of truth over a base theory $B$ is obtained by extending $B$ with the schema $\mathcal{L}_{\mathrm{B}}-\mathrm{TB}$ of $T$-biconditionals for $\mathcal{L}_{\mathrm{B}}$ :

$$
\mathcal{L}_{\mathrm{B}-\mathrm{TB}}: \quad \quad T\ulcorner\sigma\urcorner \leftrightarrow \sigma, \text { for all } \mathcal{L}_{\mathrm{B}} \text {-sentences } \sigma,
$$

where $\ulcorner\sigma\urcorner$ denotes a code (or a name) of an $\mathcal{L}_{\mathrm{B}}$-sentence $\sigma$. Now, some base theories, such as PA, contain axiom schemata, such as the schema of arithmetical induction,

\footnotetext{
7 If we formulate a theory of truth in terms of the truth predicate $T$ over a base theory B whose language $\mathcal{L}_{\mathrm{B}}$ does not contain enough names for the objects of the domain of discourse of $\mathrm{B}$, then we sometimes need a stronger theory of syntax than the ordinary finitary one, which needs to encode an expanded language $\mathcal{L}_{\mathrm{B}}^{\infty}$ with constant symbols for all objects of the domain of discourse; a typical example of such a case is

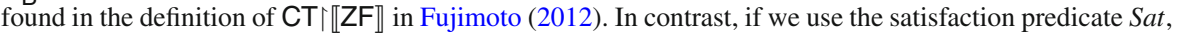
we only need to assume that base theories interpret some fixed weak fragment of arithmetic such as $I \Sigma_{1}$, independently of our choice of subject matter, and thereby we can give a uniform treatment to theories of truth across different subject matters. There is, however, a subtle technical difference between satisfaction and truth in the current axiomatic setting (see Enayat and Visser 2015), but, as far as the philosophical arguments in this article are concerned, we need not bother about it and we can assume without loss of generality that truth and satisfaction can be defined in terms of each other.
} 
but no instance of the axiom schemata containing $T$ is added to $\mathrm{TB} \uparrow$ as an axiom. So, let TB denote the extension of TB $\uparrow$ obtained by extending all the axiom schemata of $\mathrm{B}$, if any, to the entire language $\mathcal{L}_{\mathrm{B}}^{+}$. For an important example, let us write $\mathcal{L}$-Ind for the schema of arithmetical induction for a language $\mathcal{L}$ extending the language $\mathcal{L}_{\mathbb{N}}$ $\left(=\mathcal{L}_{\mathrm{PA}}=\{0, S,+, \times,<\}\right)$ of first-order arithmetic, i.e.,

$$
\mathcal{L} \text {-Ind: } \quad \varphi(0) \wedge \forall x(\varphi(x) \rightarrow \varphi(S x)) \rightarrow \forall x \varphi(x), \quad \text { for all } \varphi \in \mathcal{L} ;
$$

then the theory TB over PA is obtained from TB $\uparrow$ over PA by adding $\mathcal{L}_{\mathbb{N}}^{+}$-Ind. We secondly consider compositional theories $\mathrm{CT} \uparrow$ and $\mathrm{CT}$ of typed truth. The theory $\mathrm{CT} \uparrow$ over a base theory $B$ is obtained by augmenting $B$ with the axioms expressing the inductive clauses of the Tarskian definition of truth, such as 'for all $\mathcal{L}_{\mathrm{B}}$-sentences $\sigma$, $\neg \sigma$ is true iff $\sigma$ is not true'; see (Ketland 1999, pp. 79-80) or (Halbach 2010, p. 65) for more formal details. The crucial difference between $\mathrm{CT} \uparrow$ and $\mathrm{TB} \uparrow$ is that each truth axiom of $\mathrm{CT} \uparrow$ involves quantification over all sentences and states some property of truth about all sentences at once, whereas each truth axiom of $\mathrm{TB} \uparrow$ is an instance of the schema $\mathcal{L}_{\mathrm{B}}$-TB and only refers to a single sentence. It is known that TB $\uparrow$ is a sub-theory of $\mathrm{CT} \uparrow$ over any base theory, but the converse does not hold. ${ }^{8}$ The theory CT is obtained from $\mathrm{CT} \uparrow$ by extending all the axiom schemata of $\mathrm{B}$, if any, to $\mathcal{L}_{\mathrm{B}}^{+}$; e.g., CT over PA is defined as CT $\uparrow$ over PA plus $\mathcal{L}_{\mathbb{N}}^{+}$-Ind. In what follows, when we consider a theory of truth over a particular base theory, we indicate it by putting it in

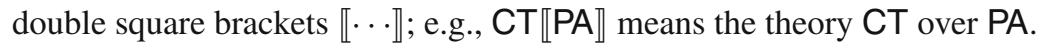

The theory $\mathrm{TB} \uparrow$ is often seen as sufficient for capturing the aforementioned two functions of truth (e.g., Halbach 1999). However, some deflationists think that TB $\uparrow$ is insufficient and $\mathrm{CT} \uparrow$ needs to be part of any adequate deflationary theory of truth. ${ }^{9}$ In fact, the schema $\mathcal{L}_{\mathrm{B}}$-TB alone does not license us to make deductions of a certain type that we expect to be able to make by means of truth. Consider the following deductive inference. ${ }^{10}$

(a) What Karl said at the trial is true.

(b) Judy said that what Ikoma said contradicts what Karl said.

(c) Therefore, if what Judy said is true, then what Ikoma said is false.

Let $K x, J x$, and $I x$ be predicates that give definite descriptions of what Karl, Judy, and Ikoma said respectively, and let $x, y$, and $z$ be the unique sentences such that $K x$, $J y$, and $I z$. Here, we need not explicitly specify what sentences Karl and Ikoma said.

\footnotetext{
8 In fact, TB $\uparrow$ can prove no general theorem with quantification over all $\mathcal{L}_{\mathrm{B}}$-sentences (Halbach 1999 , Proposition 1); e.g., the statement 'all $\mathcal{L}_{\mathrm{B}}$-sentences are either true of false' is provable in $\left.\mathrm{CT}\right\rceil$ but in neither TB $\lceil$ nor TB.

9 Field (1999, p. 535) writes 'it is more interesting to add truth in a way that includes the general laws [the axioms of CT门], since I think it is clear that without such general laws the truth predicate would not serve its main purpose.' Azzouni (1999, p. 542) seems to share the same view.

10 We assume here that Karl, Judy, and Ikoma uttered exactly one sentence. Even if they uttered more than one (but finitely many) sentences, we can still carry out the same kind of blind deduction by means of the axioms of CT $\uparrow$ by taking the conjunctions of what they uttered. However, blind deductions involving infinitely many sentences seem to need a different treatment and some further truth-theoretic axioms stronger than those of $\mathrm{CT} \uparrow$; I will expand on this point in fn 20; this point is also related to fn 12 below.
} 
Now, suppose (a) and (b). Then, what Judy said $(=y)$ is that what Ikoma said $(=z)$ implies the negation of what Karl said $(=x)$; namely, we have $y=z \rightarrow \neg x$, where we write $h$ for a representation (or a code) of a syntactic operation $h$ (see Halbach 2010, p. 32). Further suppose what Judy said is true, that is, $T y$. By the above equation, we have $T(z \rightarrow \neg x)$. From this, we want to deduce $T z \rightarrow \neg T x$, by which we immediately obtain $\neg T z$ because we have supposed (a), i.e., $T x$. However, we do not know exactly what Karl and Ikoma said; we are only given their definite descriptions and certain objects that satisfy the descriptions. In order to use $\mathcal{L}_{\mathrm{B}}$-TB in a deduction, we need to explicitly specify a sentence $\sigma$ to which we apply the schema $\mathcal{L}_{\mathrm{B}}-\mathrm{TB}$. Hence, without knowing exactly what Karl and Ikoma said, the schema $\mathcal{L}_{\mathrm{B}}$-TB cannot be used for deducing $T z \rightarrow \neg T x$ from $T(z \rightarrow \neg x)$. ${ }^{11}$ Therefore, we cannot implement the desired deduction in TB $\uparrow$ nor $T B$, and we would need some general principles such as 'for all $\mathcal{L}_{\mathrm{B}}$-sentences $v$ and $w, v \rightarrow w$ is true if and only if the truth of $v$ implies the truth of $w^{\prime}$. In this example, we give a deductive argument about the truth of some sentences by analysing and manipulating their logico-syntactic structures without explicitly specifying exactly what these sentences are. Let us call this type of deductive reasoning blind deduction. ${ }^{12}$ Neither TB $\uparrow$ nor TB enables us to carry out blind deduction in general, and the axioms of CT $\uparrow$ or something equivalent are necessary. Hence, in what follows, I assume that the axioms of CT $\uparrow$ are a minimal requirement for adequate theories of truth, and thus any adequate deflationary theory of truth must prove the axioms of $\mathrm{CT} \uparrow$.

Another function or feature that may well be required of truth is self-applicability. Suppose Karl said 'Everything the Pope says is true' and the Pope said 'Everything Judy says is true'. Then what Karl said should entail whatever Judy says. To formally implement this reasoning in a theory of truth, the theory should contain some axioms that enable iterative application of the truth predicate, since what Karl said entails what Judy says by way of what the Pope said, which involves the truth predicate. Truth-theorists ultimately have to settle the problem of what axioms are adequate for self-applicable truth, but this problem is far from settled and beyond the scope of this article, and so let us restrict our discussion to non self-applicable ('typed') truth; it is to be noted that the above example of blind deduction does not require any selfapplication of truth for the argument to go through, and we can simply assume that what Karl, Judy, and Ikoma said do not contain the truth predicate; hence, even with this restriction, we still need the axioms of CT $\uparrow$ as a minimal requirement for adequate theories of truth.

\section{Two conservativeness arguments}

I will introduce two variants of the conservativeness argument that are not vulnerable to the existing objections in the literature. The conclusion I will draw in the subsequent

\footnotetext{
11 Formally speaking, assuming sentences are coded by natural numbers, we cannot exclude the possibility that what Karl and Ikoma said are coded by non-standard natural numbers.

12 A more sophisticated example of blind deduction is the standard proof of GRef PA (see Sect. 4.2) in CT $\llbracket P A \rrbracket$, in which we reason about the truth of arbitrary sentences without explicitly specifying what they are; but, note that this deduction is made not solely by truth but also by means of inductive generalisation on numbers using $\mathcal{L}_{\mathbb{N}}^{+}$-Ind.
} 
sections is that both arguments are hard to counter if one confines one's attention to theories of truth over arithmetic, but the difficulty they pose to deflationism can be overcome by turning to other kinds of bases, which gives a support and motivation to my proposal.

\subsection{Induction as a syntactic principle}

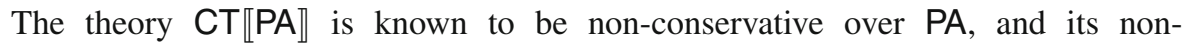
conservativeness is often considered by proponents of the conservativeness argument

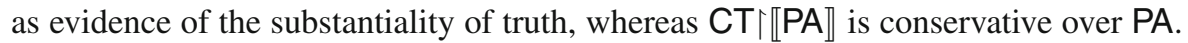
The difference between $\mathrm{CT}\lceil\llbracket \mathrm{PA} \rrbracket$ and $\mathrm{CT} \llbracket \mathrm{PA} \rrbracket$ lies in whether the schema of arithmetical induction is restricted to $\mathcal{L}_{\mathbb{N}}$ or extended to $\mathcal{L}_{\mathbb{N}}^{+}$. Hence, those proponents need to establish that an adequate theory of truth over PA must prove the schema $\mathcal{L}_{\mathbb{N}}^{+}$-Ind of arithmetical induction for the extended language $\mathcal{L}_{\mathbb{N}}^{+}$.

A paradigmatic example of such an argument to this end is proposed by Shapiro and it appeals to the indefinite extensibility of arithmetical induction. Shapiro (1998) advocates the following view:

(S1) Commitment to all instances of arithmetical induction with any predicate of natural numbers constitutes our understanding of the concept of natural number, and thus the schema of arithmetical induction should be conceived as indefinitely extensible to any newly introduced predicate of natural numbers. ${ }^{13}$

This leads him to make the next general claim:

(S2) Any adequate theory of truth over B must prove $\mathcal{L}_{\mathrm{B}}^{+}$-Ind, whenever the subject matter of $B$ includes arithmetic.

In his rejoinder to Shapiro, Field (1999) points out that (S1) does not imply (S2). His argument relies on the next two theses:

(F1) One should not conclude from the non-conservativeness of a theory of truth $\mathrm{S}=\mathrm{B}+\mathcal{T}$ over $\mathrm{B}$ that truth is substantial without first showing that each truth axiom of $\mathcal{T}$ is 'essential to truth' and postulated solely by virtue of the nature of truth.

(F2) The indefinite extensibility of the axiom schemata of the base theory B, if it is required by anything about the non-semantic subject matter of $B$, is not part of the deflationary concept of truth, and the extension of any of them is not an axiom 'essential to truth'.

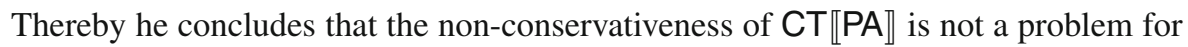
deflationists, since the indefinite extensibility at issue is derived from 'something about our idea of natural numbers' and 'nothing about truth' (p. 539). ${ }^{14}$

\footnotetext{
13 This view itself is shared by many philosophers of mathematics, such as Dummett, Parsons, and Lavine. Field (1999) also agrees with (S1) but rejects (S2) for the reason explained below.

14 Field's insight that we can generally meet the conservativeness requirement $(\mathrm{C} 1)$ by excluding the extensions of mathematical schemata, such as $\mathcal{L}_{\mathbb{N}}^{+}$-Ind, from the principles 'essential to truth' is actually
} 
There is, however, another route to (S2), not by way of (S1), which evades Field's argument. Sentences and formulae of a formal language are recursively defined, and induction on the construction (or complexity) of them is an indispensable theoremproving tool in meta-mathematics. Let us call inductive inference along the recursive construction of the syntactic structure of a formal language syntactic induction. Syntactic induction may be formulated in different ways, but a typical example of its formulation is:

(SI) Let $\Phi$ be any predicate of formulae. Suppose that $\Phi$ holds for all atomic formulae, and that if $\Phi$ holds for all sub-formulae of $A$, then $\Phi$ holds for $A$. Then $\Phi$ holds for all formulae.

In general, the way we understand how the sentences of a language are constructed is essentially of this inductive nature, and it naturally commits us to syntactic induction like (SI). ${ }^{15}$ One can thereby argue that the indefinite extensibility of syntactic induction to any newly introduced predicate of syntactic objects constitutes our understanding of the syntactic structure of any formal language no less than the indefinite extensibility of arithmetical induction constitutes our understanding of natural numbers. Therefore, whenever we use a theory of syntax for any purpose, we are committed to the indefinite extensibility of syntactic induction.

Now, recall that truth is a logico-linguistic predicate operating on syntactic objects and every theory of truth must accompany an appropriate theory of syntax. Hence, our commitment to the indefinite extensibility of syntactic induction is made prior to having any particular theory of truth and independently of any epistemic and/or mathematical commitment that we might make as to its base theory and subject matter. This suggests that the schema of syntactic induction, such as (SI), for the entire language of theories of truth is a necessary part of any adequate theory of truth; it is not essential only to truth but essential to truth and its necessary companion. Further recall that we have assumed (Syn), which says that every base theory must contain an appropriate theory of syntax on which truth operates. In particular, when a base theory B is arithmetical, a theory of syntax needs to be embedded in B via Gödel numbering, and thus induction on natural numbers and induction on syntactic objects are inevitably 'entangled' within B. If we simply identify these two induction principles, an adequate theory of truth over PA should include CT $\llbracket \mathrm{PA} \rrbracket$ and non-conservativeness thus results.

Our deflationist might try to avoid this non-conservativeness consequence by only postulating the schema of syntactic induction for $\mathcal{L}_{\mathbb{N}}^{+}$, in the form of (SI) or similar,

\footnotetext{
Footnote 14 continued

well supported by formal results obtained so far. Most, if not all, of the axiomatic theories of truth over arithmetic presented so far become conservative when we restrict arithmetical induction to $\mathcal{L}_{\mathbb{N}}$. The same applies to those over set theory or many other subject matters; for instance, if we restrict the axiom schemata of set theory to $\mathcal{L}_{\in}$, the language of first-order set theory, then the resulting theories of truth are also conservative in many cases (see Fujimoto 2012).

15 This is not only the case for formal languages but also for natural languages, although a theory of syntax for a natural language would involve more complicated simultaneous recursive definitions of the parts of speech. In his program, Hilbert took such an inductive nature of syntactic structures as the basis for his meta-mathematics and proof theory therein (see Sieg 1999).
} 
as a distinct and separate principle from $\mathcal{L}_{\mathbb{N}}^{+}$-Ind, without identifying them. However, natural numbers and syntactic objects are so intimately and deeply entangled in arithmetical base theories that the schema of syntactic induction actually implies the schema of arithmetical induction; for instance, the schema (SI) for $\mathcal{L}_{\mathbb{N}}^{+}$implies $\mathcal{L}_{\mathbb{N}}^{+}$-Ind. ${ }^{16}$ Even if we postulate the schema of syntactic induction in a form other than (SI), the schema $\mathcal{L}_{\mathrm{B}}^{+}$-Ind is still implied thereby in most cases. ${ }^{17}$ This is a quite general phenomenon, as is expected from the folklore view that the theory of syntax is essentially the same thing as arithmetic. ${ }^{18}$ Hence, in particular, CT $\llbracket \mathrm{PA} \rrbracket$ plus the

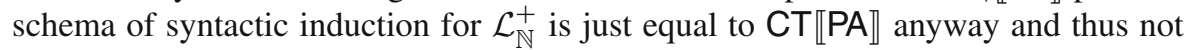
conservative over PA.

This is what I call the syntactic conservativeness argument: it aims at establishing (S2), not on the basis of the indefinite extensibility of arithmetical induction, but on the basis of that of syntactic induction. Field's theses (F1) and (F2) do not seem to help us evade this variation of the conservativeness argument, since the extension of syntactic induction to $\mathcal{L}_{\mathrm{B}}^{+}$is not required by something about the non-semantic subject matter of the theory of truth in question but rather required by the constitutive element of our understanding of the syntactic structure of formal languages.

\subsection{Commitment to logic}

One major issue concerning the logico-linguistic function of truth, in the context of the conservativeness argument, is whether truth should not only express infinite conjunctions but also establish some of them. Let $\operatorname{Bew}_{\mathrm{B}}(x)$ be a canonical provability predicate for $\mathrm{B}$ expressing ' $x$ is an $\mathcal{L}_{\mathrm{B}}$-sentence provable from $\mathrm{B}$ '. The so-called global reflection principle $G R e f_{\mathrm{B}}$ for a base theory B denotes an $\mathcal{L}_{\mathrm{B}}^{+}$-sentence $\forall x\left(\operatorname{Bew}_{\mathrm{B}}(x) \rightarrow T x\right)$, which expresses that all theorems of $\mathrm{B}$ are true. This $G R e f_{\mathrm{B}}$ is a principal example of an infinite conjunction that is claimed by proponents of the conservativeness argument to be a necessary consequence of any adequate theory of truth. They then conclude that truth is substantial, since GRef $f_{\mathrm{PA}}$ implies the consistency statement Con(PA) for PA in the presence of $\mathcal{L}_{\mathrm{B}}$-TB.

According to deflationism, the main point of having a truth predicate is that it increases one's expressive power via its logico-linguistic function. When we introduce a new expression into our vocabulary, we usually do not expect that its introduction by itself brings about any new substantive knowledge. From the deflationist point of

16 The idea of the proof of this claim is, roughly, to construct a one-to-one matching between each $n \in \mathbb{N}$ and, say, the conjunction of $n$-many $\ulcorner 0=0\urcorner$. To carry out this proof, the base theory $\mathrm{B}$ only needs to include $I \Sigma_{1}$.

17 For other examples, $\mathcal{L}_{\mathrm{B}}^{+}$-Ind is implied by the schema of induction on strings of alphabets, such as $C^{n}-I_{F O}$ in Ganea (2009) (in terms of Gödel numbering), and also by the schema of induction on the construction of sentences.

18 A variety of equivalence results are known between theories of natural numbers and syntax. Some 'pure' theories of syntax, such as Grzegorczyk's theory of concatenation and adjunctive set theory, are mutually interpretable with Q; see Švejdar (2009) and Montagna and Mancini (1994); furthermore, the results of augmenting them with the schema of syntactic induction become equivalent to PA; see Ganea (2009). Also, from the model-theoretic viewpoint, Corcoran et al. (1974) showed that the structure of strings on a finite alphabet is essentially the same as that of natural numbers. 
view, therefore, truth enables us to express infinite conjunctions, but it is not part of a deflationary theory of truth to verify or refute each given infinite conjunction, no matter how obvious its truth or falsity is. Hence, our proponent of the conservativeness argument has to provide a special reason why that particular infinite conjunction $G R e f_{\mathrm{B}}$ should be established by every adequate (deflationary) theory of truth.

I will first consider one key example of an argument for this claim and refute it, by which I intend to give a preliminary picture of what kind of infinite conjunction need not be a consequence of an adequate deflationary theory of truth. The argument I will consider is a variation of Ketland's 'reflective argument' (2005). The key thesis behind it is Feferman's influential view on 'implicit commitment': if one accepts a mathematical theory $\mathrm{S}$, then one is implicitly committed to accepting a number of further statements, such as $\operatorname{Con}(\mathrm{S})$ and proof-theoretic reflection principles for S, that are not provable in S (e.g., see Feferman 1991). Following Ketland, let us call the 'further statements' to which one is implicitly committed in accepting $S$ the reflective consequences of $\mathrm{S}$. Then, the argument goes that any adequate theory of truth must derive the reflective consequences of its base theory $B$ that are expressible in the language $\mathcal{L}_{\mathrm{B}}^{+}$and that $G R e f_{\mathrm{B}}$ is indeed among those reflective consequences. However, Field's thesis (F1) provides an immediate deflationist rebuttal to this type of argument. Any implicit commitment to a reflective consequence of an initially accepted theory is not something required by virtue of truth but rather required by one's very acceptance of the theory and one's specific epistemic and/or mathematical attitude toward the theory and/or its subject matter. Hence, the reflective consequences of any given base theory and/or its subject matter is not 'essential to truth', and thus it follows from (F1) that any non-conservativeness they cause does not undermine deflationism. In conclusion, the 'reflective argument' fails to establish that $G R e f_{\mathrm{B}}$, or any other reflective consequence such as 'all axioms of B are true', ought to be a consequence of an adequate theory of truth over B.

I have so far argued that it is not required of a deflationary theory of truth, say $\mathrm{S}$, to prove some statement $P$ solely on the ground that $P$ is a reflective consequence of the base theory $\mathrm{B}$ of $\mathrm{S}$. However, this does not exclude the possibility that the provability of some reflective consequences turns out to be necessary part of $S$ for some reason other than their being reflective consequences of B. Now, it seems still possible and reasonable to argue that a certain positive commitment to the logic one employs is a precondition for formulating any theory B of one's subject matter and any theory of truth over B, regardless of one's mathematical and/or epistemic attitude to B and its subject matter, and there may be a non-conservative truth-theoretic principle that is required, by this commitment to logic, to be a consequence of every adequate theory of truth. ${ }^{19}$ There is one strong argument that expands on this point. First note that we can formally express the following statement in $\mathcal{L}_{\mathrm{B}}^{+}$:

$$
T-\operatorname{Val}_{\mathcal{L}_{\mathrm{B}}}: \quad \text { All logically valid } \mathcal{L}_{\mathrm{B}} \text {-sentences are true. }
$$

19 In his rejoinder to Shapiro (1998), Azzouni (1999, p. 542) says that 'the capacity to establish (nonlogical!) [emphasis added] truths and generalizations about [truths of non-semantic facts] goes quite beyond what a first-order deflationist calls a deflationist theory of truth'. Hence, seemingly, he does not either exclude the possibility that even a deflationary theory of truth is required to establish some logical truths. 
Then, Cieśliński (2010) made a significant observation that GRef $f_{\mathrm{PA}}$ is equivalent over $\mathrm{CT}\left\lceil\llbracket \mathrm{PA} \rrbracket\right.$ to $T-\operatorname{Val}_{\mathcal{L}_{\mathbb{N}}} \cdot{ }^{20} \mathrm{He}$ thereby suggests that 'it is perhaps not so much the relation between truth and PA, but between truth and logic ... which matters' (p. 415). In other words, $T$ - $\mathrm{Val}_{\mathcal{L}_{\mathbb{N}}}$ is a 'reflective consequence' not of the base theory PA and/or its mathematical subject matter but rather a 'reflective consequence' of logic, to which one is committed prior to having any theory of truth or anything else.

Cieśliński's result may well give our deflationist a compelling reason to take the provability of GRef $f_{\mathrm{PA}}$ as among the essential requirements for her theory of truth over PA. Field's and other existing deflationist counterarguments seem unable to cope with this variation of the conservativeness argument, which supports (C2) by appealing to the non-conservativeness of some principle concerning logic. However, the proof of Cieśliński's theorem is very peculiar to arithmetic and does not apply to theories of truth over other subject matters, since it crucially depends on the fact that each instance $\varphi(\overline{0}) \wedge \forall x(\varphi(x) \rightarrow \varphi(x+1)) \rightarrow \varphi(\bar{n})$ on the numerals $\bar{n}$ of $\mathcal{L}_{\mathbb{N}}$-Ind is logically valid, but we do not expect the same for other mathematical axiom schemata such as the collection schema of set theory. In fact, as we will see in Sect. 6, the principle $T$-Val $\mathcal{L}_{\mathrm{B}}$ adds no substance to reasonably rich set-theoretic base theories $\mathrm{B}$, and thus $T$-Val $\mathcal{L}_{\mathrm{B}}$ is not equivalent to $G R e f_{\mathrm{B}}$ in general; furthermore, $T$-Val $\mathcal{L}_{\mathcal{L}_{\mathrm{B}}}$ does not even imply the statement 'all axioms of B are true' in general.

Let me summarise the argument in this section. Both the syntactic conservativeness argument and Cieśliński's argument point to certain general conditions for adequate theories of truth derived from consideration of our ex-ante commitment in having any theory of truth regardless of our choice of subject matter and base theory. This is why Field's and other existing defences of deflationism are unable to cope well with the two arguments, since these are designed only to avoid the requirements for non-conservative truth-theoretic principles that flow from our ex-post commitment concerning an already chosen particular subject matter and/or base theory.

20 Cieśliński (2010) also showed that GRef $\mathrm{PA}$ is equivalent, modulo CT $\uparrow \mathrm{PA} \rrbracket$, to the principle 'truth is closed under provability over $\mathcal{L}_{\mathbb{N}}$ '. Let us call this principle $T$-Cls $\mathcal{L}_{\mathrm{B}} ; T$-Cls $\mathcal{L}_{\mathrm{B}}$ obviously implies

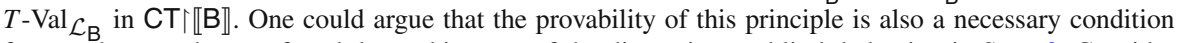
for an adequate theory of truth by making use of the discussion on blind deduction in Sect. 3. Consider the following argument: all that Karl believes are true; Judy said that what Karl believes contradicts what Ikoma said; therefore, if what Judy said is true, then what Ikoma said is false. Let us assume that Karl has infinitely many beliefs; one may well assume that he believes all (infinitely many) axioms of PA. The first premise entails the truth of infinitely many sentences; in symbolism, this is expressed as $\forall x(B x \rightarrow T x)$, where $B$ characterises the set of all sentences that Karl believes. In order to express that the infinitely many sentences, which Karl believes, contradict what Ikoma said, we need to resort to something like the provability predicate, and what Judy said would be thereby expressed as 'the negation of what Ikoma said is provable from the set of all sentences that Karl believes': in symbolism, this is expressed as $\operatorname{Bew}_{B}(\neg z)$, where $z$ is the sentence Ikoma uttered; note that $\operatorname{Bew}_{B}(\neg z)$ is an $\mathcal{L}_{\mathrm{B}}$-expression, and thus it is equivalent to the truth of what Judy said. Then, in order to carry out the blind deduction at stake, we would need the principle $T$-Cls $\mathcal{L}_{\mathrm{B}}$ to deduce the falsity of $z$ from $\operatorname{Bew}_{B}(\neg x)$ and $\forall x(B x \rightarrow T x)$. Hence, a principle like $T$-Cls $\mathcal{L}_{\mathrm{B}}$ seems necessary for a theory of truth to enable us to carry out the kind of blind deduction in question. 


\section{Theory of syntax and arithmetic}

In this section, I will discuss the legitimacy and significance of the assumption of (Syn) through examination of a recently proposed new type of theory of truth.

When a theory of truth is formulated over an arithmetical base theory, the base theory has to play two different roles at the same time, i.e., the roles of a theory of arithmetic and a theory of syntax. The equivalence of syntactic and arithmetical inductions in theories of truth over arithmetic, discussed in Sect. 4.1, comes from this very entanglement of the two roles within a single theory. This is an inevitable consequence under the assumption of (Syn). By contrast, in our ordinary informal meta-mathematical discourse, the theory of syntax and that of natural numbers are kept separate, and syntactic objects and natural numbers are treated as distinct objects.

Having reflected upon this dissonance between the customary methodology in axiomatic theories of truth and our informal meta-mathematics, Heck (2009), Halbach (2010, Ch. 21) and Leigh and Nicolai (2013) proposed a new type of theory of truth in which a theory of syntax is given as a completely separate theory from the base theory $\mathrm{B}$, with a new domain of its own objects separate from the domain of the non-semantic objects of $\mathrm{B}$. This new type of theory of truth is quite versatile and can be applied to literally any formal theory of any subject matter, whether or not it is rich enough to develop a theory of syntax within it. Hence, the universality of truth, discussed in Sect. 2, is better captured by this formal setting than our current one with the exclusive condition (Syn). More importantly, theories of truth of this type are always conservative over their base theories even in the presence of the extended syntactic induction as well as Cieśliński's principle in terms of the separate 'disentangled' theory of syntax. ${ }^{21}$ This general conservation result indicates that (Syn) is a crucial and indispensable assumption implicit in the conservativeness argument: indeed, all the arguments for the claim (C2) presented so far in the literature are only valid under the assumption of (Syn) and, if we adopt theories of truth with a disentangled theory of syntax, conservativeness results in all the known relevant cases. So, one easy way out for deflationists from the predicament at issue, posed by the syntactic conservativeness argument and Cieśliński's argument, is to abandon the assumption of (Syn) by taking up this new formal conception of 'theory of truth'. However, this is not a genuine solution to the problem. Theories of truth of this type with a disentangled theory of syntax turn out to be unnatural and inappropriate when we think of the ultimate goal of theories of truth. ${ }^{22}$

A primary purpose of the axiomatic approach to truth is to provide a minimal framework for implementing the notion of truth onto a maximally rich theory in the sense that we do not want to ascend beyond it to a meta-language and a meta-theory

\footnotetext{
21 With a theory of syntax completely separate from a theory of natural numbers, we can make a clear formal distinction of arithmetical induction and syntactic induction in this new framework. Then we can show that the compositional theory of typed truth over B with a disentangled theory of syntax, written as $C T D[\mathrm{~B}]$ in Leigh and Nicolai (2013), is conservative over B even with the addition of the full schema of syntactic induction for the entire language: the same holds for the principles corresponding to Cieśliński's $T-\operatorname{Val}_{\mathcal{L}_{\mathrm{B}}}$ and $T$-Cls $\mathcal{L}_{\mathrm{B}}$.

22 Halbach (2010, p. 320) also draws the same conclusion for a reason similar to mine.
} 
even richer than it in non-semantic content. A typical example of a maximally rich theory is the theory within which one carries out all her mathematical investigation. For instance, suppose one wants to give a theory of truth over the theory M of one's entire mathematics. If she wants to define a truth predicate over $\mathrm{M}$ by mathematical means, she has to ascend to some meta-theory richer in mathematical content than $M$ due to Tarski's theorem; for example, if M is ZF, then she has to ascend to some richer theory, such as the Morse-Kelly theory MK of classes, to define the truth over ZF. In this situation, one would prefer to dispense with ascent to any such meta-theory, as otherwise her mathematics would be enlarged by the new mathematical content of such a meta-theory and thus $\mathrm{M}$ would no longer be the theory of her entire mathematics. Here is the point where the axiomatic approach to truth comes into play: we introduce truth as an undefined primitive predicate and also as a non-mathematical logico-linguistic notion, and then directly characterise it by listing its axioms, which requires no addition of mathematical substance. ${ }^{23}$ Another example of a maximally rich theory is the theory W of one's entire (non-semantic) science, say, the conglomerate of one's current best theories of mathematics, physics, chemistry, and so forth; then, the subject matter of this theory is everything she investigates in science and its language is the (nonsemantic) part of her natural language used in her scientific discourse. Surely, she would not like to change her theory of physics or any other scientific discipline only for the sake of having a truth predicate for her natural language. Most philosophers, I think, are ultimately interested in the theory of truth for such maximally rich theories and subject matters and, as the above examples indicate, some of them, such as M and W, are naturally presumed to be indeed quite 'rich' so that they intrinsically contain a theory of syntax per se and even develop substantial meta-mathematics on the basis of the theory of syntax therein. ${ }^{24}$

Now, for such 'rich' theories, adding another theory of syntax separately from the existing theory of syntax intrinsically contained in them is quite unnatural and even unsound. In the case of theories of truth with a disentangled theory of syntax, many syntactic statements, such as the consistency statement for the base theory B, are provable in terms of the newly added theory of syntax, but those statements are not provable in terms of the intrinsic theory of syntax of B; namely, the two theories of syntax behave differently and have incompatible consequences. Hence, while the assumption of (Syn) is not necessary and theories of truth with a disentangled theory of syntax behave ideally for deflationists in many cases, where base theories are relatively weak ('poor'), the assumption of (Syn) is still indispensable and those theories with

\footnotetext{
23 Väänänen's (2001) notion of urlogic can be regarded as the theory of our entire mathematics that is not treated as an object of meta-mathematics such as model theory, and so it gives another important example of a maximally rich theory; also see fn 2 .

24 Some philosophers and mathematicians suspect that those very 'rich' theories such as ZF lack foundational justification, and may refuse to accept them even as a maximally rich theory of their mathematical investigation. For them, even a maximally rich theory may not be 'rich' in the sense at issue. In an extreme case, one might only accept pure arithmetic as real mathematics; e.g., Hilbert's finite standpoint. However, as I will conclude below, the conservativeness argument limited to theories of truth over arithmetic cannot achieve its goal of undermining deflationism anyway. The issue would be more subtle when one takes an intermediate theory, which is richer than arithmetic but still not 'rich' in the sense at issue, as one's foundation of mathematics; e.g., a weak predicative fragment of second-order arithmetic. This case will be considered in Sect. 7 to some extent.
} 
a disentangled theory of syntax are unnatural and inappropriate in other cases, where base theories are 'rich'. ${ }^{25}$ The consideration of maximally rich theories indicates that there is indeed a case where theories of truth with a disentangled theory of syntax are not appropriate and we really need to pursue axiomatic theories of truth over a 'rich' base theory with the assumption of (Syn). Hence, theories of truth with a disentangled theory of syntax do not provide a general solution to the dilemma posed by the conservativeness argument, when we take a broader range of subject matters and theories into account as the bases of theories of truth.

\section{Beyond arithmetic}

Now we have arrived at the main section of this paper, and I will elaborate on my proposal.

The preceding discussion of the assumption of (Syn) gives a new perspective to the debate on the conservativeness argument. It is theories of truth over 'rich' subject matters and base theories that essentially need the assumption of (Syn). A typical example of such a 'rich' subject matter (in mathematics) is set theory, but arithmetic is deemed to be not 'rich' enough in the sense at issue. Arithmetic does not treat syntactic objects as its intended subject matter, and any mathematical theory about or built up on the basis of those syntactic objects is not part of arithmetic per se. So, the debate on the conservativeness argument so far has been misplaced in an atypical formal setting for the assumption of (Syn).

Does the non-conservativeness of some theories of truth obtained in the 'atypical' setting, where these theories of truth are formulated over arithmetic with the assumption of (Syn), still imply the substantiality of truth? My answer is no. According to the aforementioned folklore view, the mathematical structure that the theory of syntax describes is essentially the same as the mathematical structure of natural numbers, and thus arithmetic is indeed a minimal basis for theories of truth under the assumption of (Syn). An arithmetical base theory B is at the same time a theory of syntax, and the non-semantic base content and the syntactic content of a theory of truth over B become almost identical. With this 'entanglement', a theory of truth over arithmetic can then be seen as having little or no substantial non-semantic content to which truth is

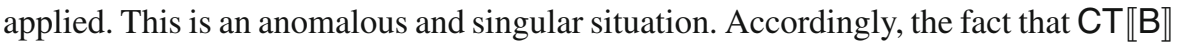
is not conservative over an arithmetical base theory $\mathrm{B}$ is interpreted to mean that truth is not conservative over a theory of syntax, but this is not a problem for deflationism, because truth axioms and a theory of syntax always come in one package and it makes little sense to separate and compare them in terms of conservativeness. Even if we somehow separate and compare them, truth is a logico-linguistic device operating on

\footnotetext{
25 One might propose, as an alternative solution to the problem at issue, to adopt purely disquotational theories of truth like TB, instead of CT, for the sake of conservativeness in the cost of the logico-linguistic function of blind deduction; $T B \llbracket B \rrbracket$ is conservative over any base theory $B$ even if $B$ contains other axiom schemata than arithmetical induction (with the proviso that $\mathrm{B}$ contains the schemata unrestrictedly for $\mathcal{L}_{\mathrm{B}}$ ). However, the cost would be not only blind deduction. One would likely have to give up self-applicability as well, since theories of purely disquotational but self-applicable truth are often not conservative over

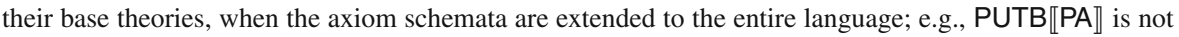
conservative over PA (see Halbach 2009).
} 
syntactic objects, and thus it would be no surprise anyway that truth adds some syntactic substance to a theory of syntax. ${ }^{26}$ Semantics always comes with syntax, and syntax is not among the non-semantic subject matters on top of which deflationary truth should be conservatively added; non-conservativeness over syntax is not what deflationists would take as a sign of the substantiality of truth. ${ }^{27}$

Having reflected upon the fundamental motivation for (Syn) and observed the singularity of theories of truth over arithmetic, I now propose that we should exclude arithmetic (and other 'poor' subject matters) from the range of the subject matters to be taken into account in (C1) and we should turn to 'richer' subject matters for evaluating the conservativeness argument. Our proponent of the conservativeness argument has no choice but to accept this proposal, since the conservativeness argument based solely on the formal results of theories of truth over arithmetic cannot undermine deflationism: on the one hand, if theories of truth over arithmetic is given with an embedded theory of syntax via coding and syntactic induction entangled with arithmetical induction, then the non-conservativeness of them does not imply the substantiality of truth; on the other hand, if they are formulated with a disentangled theory of syntax and syntactic induction in terms of it, then the conservativeness requirement (C1) is generally met and thus the non-conservativeness claim (C2) simply fails. In contrast to arithmetic, some 'rich' subject matters do have substantially rich non-semantic content besides its syntactic content and intrinsically contain a theory of syntax per se; hence, the above argument against the conservativeness argument cannot be generalised to the case where such a 'rich' subject matter is taken as the basis of theories of truth. Among others, set theory is a typical example of such a 'rich' subject matter: it is often taken as the foundation of mathematics and many would consider some standard theories of sets, such as ZF, to be maximally rich in the aforementioned sense; also, a theory of syntax per se is regarded as an intrinsic part of set theory, and set theory is rich enough to develop substantial meta-mathematics on the basis of the theory of syntax therein.

Now, let us turn to consider theories of truth over set theory with the assumption of (Syn). Recall that the crucial difference at issue between arithmetic and set theory is that set theory intrinsically contains a theory of syntax and is 'rich' enough to implement substantial meta-mathematics on the basis of it. We should proceed with this difference in mind. However, as the aforementioned folklore view goes, a variety

\footnotetext{
26 Let me give an illustrative analogy in support of this point. Consider a partial propositional logic only with three connectives $\wedge, \vee$, and $\rightarrow$ together with the ordinary introduction and elimination rules for these connectives. If we add the negation $\neg$ as a new connective together with the ordinary rules for it, then we can derive new tautologies that do not contain $\neg$; e.g., Peirce's law. This fact could be described as the non-conservativeness of the negation $\neg$ over the partial propositional logic. Now, one might well argue that logical notions should not be substantial, but one would not conclude on the ground of this nonconservativeness result that the negation $\neg$ is substantial and thus not logical; these four connectives should be taken as one package and it makes little sense to separate and compare them in terms of conservativeness; also, those new logical truths are rather natural and expected consequences of the addition of the new logical device $\neg$.

27 Even if we adopt theories of truth with a disentangled theory of syntax, the same non-conservativeness results over the disentangled theory of syntax. However, this fact is not taken by proponents of those theories as a problem for deflationism either, and they also claim that it does not imply the substantiality of truth; see Nicolai (2015, Sect. 2.3).
} 
of different formulations of the theory of syntax, in terms of finite sequences, trees, natural numbers, and so forth, share essentially the same inductive structure (cf. fn 17), and set theory is rich enough to prove this fact; indeed, we usually need not distinguish different formulations of the theory of syntax in the actual practice of mathematical logic within set theory. Hence, in order to treat theories of truth over arithmetic and set theory in a uniform way, I will assume that the schema of syntactic induction, no matter how it is (reasonably) defined, is equivalent to the schema of arithmetical induction, and, in what follows, I will identify the schema of syntactic induction for $\mathcal{L}_{\in}^{+}$with $\mathcal{L}_{\epsilon}^{+}$-Ind expressed in terms of the standard translation of $\mathcal{L}_{\mathbb{N}}$ into $\mathcal{L}_{\epsilon}$, where $\mathcal{L}_{\in}\left(=\mathcal{L}_{\text {ZF }}\right)$ is the language of first-order set theory. ${ }^{28}$ Then, in sharp contrast to arithmetical base theories, many theories of truth over sufficiently strong theories of sets, such as ZF, become conservative even with the addition of $\mathcal{L}_{\epsilon}^{+}$-Ind (equivalently, the schema of syntactic induction for $\mathcal{L}_{\epsilon}^{+}$).

Theorem 1 If $\mathrm{B}$ is an $\mathcal{L}_{\in}$-theory extending $\mathrm{ZF}$, then $\mathrm{CT} \uparrow \llbracket \mathrm{B} \rrbracket+\mathcal{L}_{\epsilon}^{+}$-Ind is conservative over B. ${ }^{29}$

Proof Suppose $\mathrm{CT}\left\lceil\llbracket \mathrm{B} \rrbracket+\mathcal{L}_{\epsilon}^{+}\right.$-Ind $\vdash \sigma$ for an $\mathcal{L}_{\epsilon}$-sentence $\sigma$. Let $\mathrm{U}$ be the collection of the axioms of $\mathrm{B}$ used in the derivation of $\sigma$. By the Montague-Lévy reflection principle, B proves that there exists an admissible set $X$ such that $X$ contains the set of natural numbers $(=\omega)$, and

$$
\text { if } \neg \sigma \text {, then } X \text { is a transitive model of } U \text { and } \neg \sigma \text {. }
$$

Since $X$ is admissible, we can define the truth class (or the full satisfaction class) of $X$ and interpret the truth predicate $T$ (or the satisfaction predicate Sat) thereby. Furthermore, the transitivity of $X$ and $\omega \in X$ automatically verify $\mathcal{L}_{\epsilon}^{+}$-Ind under this interpretation. Hence, if $\neg \sigma$ were the case, the deduction of $\sigma$ in $\mathrm{CT} \backslash \llbracket \mathrm{B} \rrbracket+\mathcal{L}_{\in}^{+}$-Ind could be modeled in $X$ and thus $X$ would satisfy both $\sigma$ and $\neg \sigma$, which is impossible.

We have the same phenomenon even with a relatively weak subject matter, secondorder arithmetic, although it is debatable whether second-order arithmetic is 'rich' enough in the sense at issue. Let $Z_{2}$ denote the theory of full analysis $\left(=\Pi_{\infty}^{1}-C A\right.$, see Simpson 2009) over the language $\mathcal{L}_{\mathbb{N}}^{2}$ of second-order arithmetic. Then, the following $\operatorname{holds}^{30}$ :

\footnotetext{
28 Even if we use a theory of syntax of a language of proper size class, such as $\mathcal{L}_{\in}^{\infty}$ (see fn 7), the expressions of the language are defined essentially by $\omega$-recursion anyway and thus any reasonably defined syntactic induction for such a language is expected to be equivalent to arithmetical induction. Furthermore, also in this setting, we can show the same statement as Theorem 1 in a parallel manner.

29 This is a modification of Fujimoto (2012, Theorem 20), which shows that CT $\lceil B \rrbracket$ is conservative over $\mathrm{B} \supset \mathrm{ZF}$ even with the addition of the full separation schema for $\mathcal{L}_{\epsilon}^{+}$. This modification yields a more general conservation result: $\mathrm{CT} \backslash \llbracket \mathrm{B} \rrbracket+\mathcal{L}_{\epsilon}^{+}$-Ind is conservative over any $\mathcal{L}_{\in}$-theory $\mathrm{B} \supset \mathrm{KP} \omega+\Pi_{\infty}$-Reflection [see Rathjen (1994)]; as a matter of fact, the same holds for even weaker $\mathcal{L}_{\epsilon}$-theories such as $B=K P \omega$ but the proof requires a slightly different argument making use of the fact stated in fn 30 . Also note that the presence of urelements would not affect the statement of the theorem and would not require any substantial change of the proof either, as long as the class of urelements is set-sized.

30 Theorem 2 is shown in a completely parallel manner to Theorem 1 by using the $\omega$-reflection principle (see Simpson 2009, Lemma VIII.5.2) instead of the Montague-Lévy reflection principle. Let Bi be ACA
} 
Theorem 2 If $\mathrm{B}$ is an $\mathcal{L}_{\mathbb{N}}^{2}$-theory extending $\mathrm{Z}_{2}$, then $\mathrm{CT} \uparrow \llbracket \mathrm{B} \rrbracket+\left(\mathcal{L}_{\mathbb{N}}^{2}\right)^{+}$-Ind is conservative over $\mathrm{B}$.

These theorems tell us that, when the non-semantic content of a base theory B is 'rich' enough, the extension of syntactic induction to $\mathcal{L}_{\mathrm{B}}^{+}$does not add any nonsemantic substance. ${ }^{31}$ They also validate my claim in Sect. 4.2 that Cieśliński's argument only applies to arithmetic; for, if $\mathrm{B}$ is as in Theorems 1 or 2, then $\mathrm{CT} \uparrow \llbracket \mathrm{B} \rrbracket+\mathcal{L}_{\mathrm{B}}^{+}$-Ind proves Cieśliński's principle $T$ - $\mathrm{Val}_{\mathcal{L}_{\mathrm{B}}}$. Furthermore, the same conservation result holds for many other axiomatic theories of truth, including most (if not all) of the so far presented theories of self-applicable truth, over any base theory B satisfying the condition of Theorems 1 or $2 .{ }^{32}$ That is to say, we can conservatively add even type-free self-applicable truth to these base theories together with the full schema of syntactic induction. Hence, if we adopt those 'rich' bases, we need not give up either blind deduction nor self-applicability as the logico-linguistic functions of truth. ${ }^{33}$

Let us summarise the points I have made. First, by Field's theses (F1) and (F2), any mathematical axiom schemata of $\mathrm{ZF}$ or $\mathrm{Z}_{2}$ postulated by virtue of its subject matter, such as the collection schema of $Z F$ and the comprehension schema of $Z_{2}$, need not be extended to $\mathcal{L}_{\epsilon}^{+}$or $\left(\mathcal{L}_{\mathbb{N}}^{2}\right)^{+}$in order to obtain an adequate deflationary theory of truth; only syntactic (arithmetical, equivalently) induction is required to be extended because of its indefinite extensibility as a constitutive element of our understanding of the bearers of truth. Second, as I have argued, even if an infinite conjunction expressed in terms of $T$ is a reflective consequence of $Z F$ (or $Z_{2}$ ), the infinite conjunction, such as $G R e f_{\mathrm{ZF}}$ (or $G R e f_{\mathrm{Z}_{2}}$ ) and the statement 'all axioms of ZF $\left(\right.$ or $Z_{2}$ ) are true', need not be a consequence of an adequate deflationary theory of truth solely on the ground of its being a reflective consequence of ZF (or $Z_{2}$ ). As far as I

\section{Footnote 30 continued}

plus the schema $\mathrm{Bi}$ of bar induction; $\mathrm{Bi}$ is known to be proof-theoretically equivalent to the first-order theory of inductive definitions $I D_{1}$. Since Bi derives the $\omega$-reflection principle and is closed under $\omega$ Turing jumps, Theorem 2 can be strengthened to the following: $\mathrm{CT}\left\lceil\llbracket \mathrm{B} \rrbracket+\left(\mathcal{L}_{\mathbb{N}}^{2}\right)^{+}\right.$-Ind is conservative over $\mathrm{B}$ for any $\mathcal{L}_{\mathbb{N}}^{2}$-theory $\mathrm{B} \supset \mathrm{Bi}$.

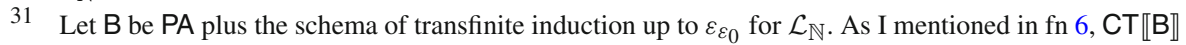
is conservative over $\mathrm{B}$. The same conservation holds even if we replace $\varepsilon_{\varepsilon_{0}}$ by many other ordinals such as $\varphi_{2} 0, \varphi_{\varepsilon_{0}} 0, \Gamma_{0}$, etc. Hence, even if $\mathrm{B}$ is an $\mathcal{L}_{\mathbb{N}}$-theory, $\mathrm{CT}\left\lceil\llbracket \mathrm{B} \rrbracket+\mathcal{L}_{\mathbb{N}}^{+}\right.$-Ind is sometimes conservative over $\mathrm{B}$, when B contains axioms of 'higher-order' and non-purely arithmetical nature in the sense of Isaacson (1987).

32 For instance, the Kripke-Feferman theories over $Z F$ and $Z_{2}$ are conservative over their bases, when only syntactic induction is extended to the whole language and all the other axiom schemata are restricted to the language of the base theory (see Fujimoto 2012).

33 The crucial point of the proofs of Theorems 1 and 2 is that the constructed transitive models and $\omega$-models keep the (first-order) arithmetical part unchanged. One might wonder why a similar proof is not possible over (first-order) arithmetical base theories. Certain types of 'reflection principles' are indeed provable in reasonably strong arithmetical theories; for instance, it is provable in PA, essentially due to the arithmetised completeness theorem and the reflexivity of PA, that if an $\mathcal{L}_{\mathbb{N}}$-sentence $\varphi$ holds then there is an arithmetised model of $\varphi$. However, the domain of an arithmetised model thus constructed may have a quite different structure from the set $\mathbb{N}$ of natural numbers, and we cannot expect the model to satisfy arithmetical induction for arbitrary formulae that may contain the truth predicate for the model. Furthermore, the truth predicate for such a model does not necessarily satisfy the axioms of CT $\uparrow$, since the formula expressing ' $x$ is an $\mathcal{L}_{\mathbb{N}}$-sentence' may have a different meaning in the model. 
know, there has been presented no argument so far that compels deflationists to accept the extension of those mathematical axiom schemata or the provability of these infinite conjunctions as necessary part of adequate deflationary theories of truth over ZF (or $Z_{2}$ ). Consequently, $C T \nmid \llbracket Z F \rrbracket+\mathcal{L}_{\mathbb{N}^{-}}^{+}$Ind and $\mathrm{CT} \uparrow \llbracket Z_{2} \rrbracket+\mathcal{L}_{\mathbb{N}}^{+}$-Ind can be (provisionally) seen as adequate theories of truth from the deflationist point of view, but they are still conservative over their base theories. By moving to 'rich' base theories, for which the condition (Syn) is appropriately applied, the problems are suddenly dissipated.

\section{The conservativeness requirement re-examined}

In this section, I will consider a possible objection to my proposal, and introduce a new issue into the debate through examination of that objection.

We should not hastily conclude that our deflationist has finally succeeded in refuting the conservativeness argument. As the next proposition shows, not all base theories of even 'rich' subject matters enjoy the same strong conservativeness property as ZF and $Z_{2}$ do.

Proposition 3 Let $\mathrm{B}$ be either an $\mathcal{L}_{\mathbb{N}^{-}}^{2}$ or $\mathcal{L}_{\epsilon}$-theory such that $\mathrm{B} \subset \mathrm{F}+\mathcal{L}_{\mathrm{B}}$-Ind for some finitely axiomatisable $\mathcal{L}_{\mathrm{B}}$-theory $\mathrm{F}$. Then, $\mathrm{CT} \backslash \llbracket \mathrm{B} \rrbracket+\mathcal{L}_{\mathrm{B}}^{+}$-Ind proves GRef $f_{\mathrm{B}}$, and thus it is not conservative over $\mathrm{B}$. The proof is standard and I omit it.

We have a similar non-conservativeness phenomenon concerning $T$-Val $\mathcal{L}_{\mathrm{B}}$, but let us focus on the indefinite extensibility of syntactic induction and its implications, such as $\mathcal{L}_{\mathrm{B}}^{+}$-Ind, for simplicity. ${ }^{34}$ For example, let $\mathrm{ZF}_{n}(n \geq 1)$ be the result of restricting the two axiom schemata of ZF, i.e., the separation and collection schemata, to the $\Sigma_{n}$-formulae in the Lévy hierarchy. It is known that $Z F_{n}$ is finitely axiomatisable. Put $\mathrm{B}=\mathrm{ZF}_{n}+\mathcal{L}_{\epsilon}$-Ind. This $\mathrm{B}$ satisfies the condition of Proposition 3. Therefore, it follows that $\mathrm{CT} \uparrow \llbracket \mathrm{B} \rrbracket+\mathcal{L}_{\epsilon}^{+}$-Ind is not conservative over $\mathrm{B}$. $^{35}$

Could one thereby form a new conservativeness argument by appealing to Proposition 3 to the effect that she has found a counterexample, say, $Z_{79}+\mathcal{L}_{\in}$-Ind, of the conservativeness requirement $(\mathrm{C} 1)$ and thus truth is substantial? As I will argue below, this non-conservativeness result does not automatically entail the substantiality of truth, and there remain more issues that one would have to discuss and settle before concluding the substantiality of truth.

Firstly, conservativeness is not always required as an adequacy condition for deflationary theories of truth. We have seen in the last section that the conservativeness requirement $(\mathrm{C} 1)$ should not be applied to theories of truth over arithmetic. Here I will further argue that it need not either be applied to theories of truth over some base theories of even 'rich' subject matters. According to the syntactic conservativeness argument, the schema $\mathcal{L}_{\mathrm{B}}^{+}$-Ind is required to be part of an adequate theory of truth by

\footnotetext{
34 Let $B$ be as in Proposition 3 and suppose $B$ further satisfies the following: (i) $\mathcal{L}_{\mathrm{B}} \supset \mathcal{L}_{\mathbb{N}}$; (ii) the $\mathcal{L}_{\mathbb{N} \text {-part }}$ of $\mathrm{B}$ includes $I \Sigma_{1}$ and plays the role of the theory of syntax for theories of truth over $\mathrm{B}$. Then, we can show, in a parallel manner to Cieśliński's original theorem, that $\mathrm{CT} \backslash \llbracket \mathrm{B} \rrbracket+T-\mathrm{Val}_{\mathcal{L}_{\mathrm{B}}}$ is not conservative over $\mathrm{B}$.

35 For another example, neither CT $\backslash \llbracket \Pi_{n}^{1}-\mathrm{CA} \rrbracket+\left(\mathcal{L}_{\mathbb{N}}^{2}\right)^{+}$-Ind nor CT $\uparrow \llbracket \Pi_{n}^{1}-\mathrm{CA} \rrbracket+T-V^{2} \mathcal{L}_{\mathbb{N}}^{2}$ is conservative for any $n \in \mathbb{N}$.
} 
our commitment to the indefinite extensibility of syntactic induction. Hence, if there is any other principle derived from the same commitment but expressible in the base language $\mathcal{L}_{\mathrm{B}}$, then we are also committed to accepting it regardless of truth. In particular, we are committed to accepting the full induction schema $\mathcal{L}_{\mathrm{B}}$-Ind for the base language $\mathcal{L}_{\mathrm{B}}$ prior to adding truth to $\mathrm{B}$. However, it is known that any finitely axiomatisable theory B cannot prove $\mathcal{L}_{\mathrm{B}}$-Ind (see Hájek and Pudlak 1993, Lemma 3.47). Hence, any finitely axiomatisable theory $B$ fails to fulfill the commitment to the indefinite extensibility of syntactic induction. ${ }^{36}$ This indicates that, if non-conservativeness is a sign of substantiality, the commitment to the indefinite extensibility of syntactic induction is already fairly substantial regardless of truth. And if such commitment is a necessary part of an adequate theory of truth, we have no good reason to require the theory of truth to be conservative over all arbitrary base theories. Furthermore, the syntactic structure of formal languages and our understanding of it might lead us to commit ourselves to some other things of substance, in addition to the indefinite extensibility of syntactic induction, which cause further non-conservativeness results; for instance, constructibility of functions and objects by recursion along $\omega$ might be considered as such. Therefore, our proponent of the conservativeness argument has to demonstrate that there is indeed a case where (1) truth is still required to be conservatively added to some base theory $B$ in spite of the substantiality of the commitment at issue concerning the syntactic structure of formal languages and (2) any adequate theory of truth over that base theory $B$ is non-conservative. This does not seem to be an easy task. For instance, if a theory B is so 'rich' as to exhaust all our mathematical commitment concerning the syntactic structure of formal languages and thereby make the commitment negligibly insubstantial, then one may well insist on (1) for such B, but we have just seen that an allegedly adequate theory of deflationary truth over some natural candidates of such very 'rich' theories satisfying (1), such as ZF, is conservative; now, it is far from clear whether there is indeed any theory B that satisfies both (1) and (2).

Secondly, we have to always ask whether the axiomatic approach to truth with the assumption of (Syn) is indeed an appropriate (or necessary) formal setting in a given context. First of all, some other type of theory of truth might be more appropriate than axiomatic theories of truth: if the axiomatic approach is not taken, the conservativeness argument is unlikely to go through (see fn 2). For instance, if one is working within set theory, then the ordinary model theory or some semantic theory of truth might be more appropriate than the axiomatic approach as the theory of truth for, say, second-order arithmetic or real analysis. Next, even when the axiomatic approach is legitimately taken, the condition (Syn) need not be assumed in all cases and one needs to examine whether the assumption of (Syn) is necessary in a given case. I argued that 'rich' subject matters essentially need the assumption of (Syn) and this was the reason why we turned

\footnotetext{
36 A similar argument can be made against Cieśliński's argument. Let B be as in fn 34 and further assume that B has partial truth (satisfaction) predicates. Consider the following 'partial realization', $T$-Val $\left.\mathcal{L}_{\mathrm{B}}\right\rceil_{k}$, of $T$-Val $\mathcal{L}_{\mathrm{B}}$ expressible in the base language $\mathcal{L}_{\mathrm{B}}$ : 'If $\sigma$ is logically valid $\mathcal{L}_{\mathrm{B}}$-sentence and its complexity is $\leq k$, then $\sigma$ is true in terms of the partial truth predicate $T_{k}$ for the sentences of complexity $\leq k$.' If we take $T$ - $\mathrm{Val}_{\mathcal{L}_{\mathrm{B}}}$ as an implication of our commitment to logic, then we would also naturally accept the $\mathcal{L}_{\mathrm{B}^{-}}$ sentences $T$-Val $\left.\mathcal{L}_{\mathrm{B}}\right\rceil_{k}$ for all $k \in \mathbb{N}$ as implications of the same commitment. Then, $\left.\mathrm{B}+\left\{T \text {-Val } \mathcal{L}_{\mathrm{B}}\right\rceil_{k} \mid k \in \mathbb{N}\right\}$ is not conservative over any finitely axiomatisable $B$.
} 
to consider theories of truth over set theory. However, when a given subject matter is not 'rich' in the sense at issue, our proponent of the conservativeness argument has to somehow demonstrate that (Syn) is still an appropriate and necessary assumption for theories of truth over that subject matter. ${ }^{37}$ Third, even if the subject matter in question is 'rich', a given base theory B may not fully capture the relevant content of the subject matter and sufficiently represent the 'richness' of it. For instance, the mere fact that the language $\mathcal{L}_{\mathrm{B}}$ of a base theory $\mathrm{B}$ is the language $\mathcal{L}_{\in}$ of set theory does not necessarily mean that $B$ fully captures the relevant content of set theory; the $\mathcal{L}_{\in}$-theory ZF is the most natural and standard axiomatisation of set theory, and it presumably fully captures all the relevant content of set theory at issue, but we can make up an $\mathcal{L}_{\epsilon}$-theory that is so weak or unnatural that we can't even take it to be a theory of sets. Here is an important difference between our new formal setting and the older customary setting in which only arithmetical base theories are considered: in the older setting, the most natural and standard (and even 'complete' according to Isaacson 1987) axiomatisation PA of arithmetic makes some allegedly adequate deflationary theories of truth non-conservative; by contrast, in our new setting, the same theories of truth over the standard theory ZF of sets are conservative; therefore, proponents of the conservativeness argument now have to search the realm of much less natural non-standard theories of the subject matter in question, such as $Z_{79}+\mathcal{L}_{\in}$-Ind, for evidence of the substantiality of truth.

All these indicate that the conservativeness requirement $(\mathrm{C} 1)$ is to be posed only to some limited range of base theories, and let us call such a base theory that falls within the proper scope of $(\mathrm{C} 1)$ an adequate basis for the conservativeness requirement ('adequate basis' for short); note that this notion is relative to what adequacy condition is set for theories of truth (and how it is justified), but let us assume that we are given some fixed such. In other words, we now reject (C3) and replace it by the following:

(C4) Whenever a theory B is an adequate basis, any adequate theory of truth over B must be conservative over $B$.

An immediate question is: what theories are counted as adequate bases? Presumably ZF is counted as such, but it is highly questionable whether the same can be said of $\mathrm{ZF}_{79}+\mathcal{L}_{\in}^{+}$-Ind. Our proponent of the conservativeness argument needs to give a non-ad hoc answer to this question in such a way that at least one adequate basis $B$ makes truth non-conservative. The burden of proof is now on the proponent, and this does not look an easy task. ${ }^{38}$

\footnotetext{
37 For instance, if one takes a relatively weak subsystem of second-order arithmetic as her foundation of mathematics, then the subsystem is maximally rich for her and the axiomatic approach to truth should be taken for it; cf. fn 24. I do not have a conclusive answer to whether the conservativeness argument would go through in such a case: the main issue here is whether or not (Syn) is necessary and (C1) is appropriately applied to such a case.

38 For instance, a finitely axiomatisable theory, say, $\mathrm{ZF}_{2016}$, has higher consistency strength and interpretability degree than $\mathrm{B}:=\mathrm{ZF}_{79}+\mathcal{L}_{\in}$-Ind, and even proves the existence of a 'standard' model of $\mathrm{CT} \backslash \llbracket \mathrm{B} \rrbracket+\mathcal{L}_{\in}^{+}$-Ind in which all the deductive aspects of that theory of truth are realised. However, $\mathrm{ZF}_{2016}$ is not an adequate basis, since it fails to fulfill the commitment to the indefinite extensibility of syntactic induction. Then, proponents of the conservativeness argument would have to give a non-ad hoc explanation of why $\mathrm{B}$ is adequate while $\mathrm{ZF}_{2016}$ is not.
} 


\section{Summary and conclusion}

Two versions of the conservativeness argument were presented in Sect. $4,{ }^{39}$ and we have seen that neither a purely disquotational theory of truth nor a theory of truth with a disentangled theory of syntax provides deflationists with a general solution to the problem raised by them: an adequate deflationary theory of truth should contain the compositional axioms of CT $\uparrow$, and there are some important cases where the condition (Syn) should be assumed. Then, I argued that the conservativeness argument solely based on the non-conservativeness of (axiomatic) theories of truth (with the assumption of (Syn)) over arithmetic nonetheless fails to undermine deflationism, and thereby concluded that we should turn to consider theories of truth over 'richer' subject matters in order to properly evaluate the success (or failure) of the conservativeness argument. However, it turned out that an adequate theory of deflationary truth is conservative over sufficiently strong theories of sets (or second-order arithmetic). This conservation result and the consideration of theories of truth over these 'richer' bases in Sect. 7 give a sharper focus to an issue that has not been sufficiently discussed so far: What are adequate bases for the conservativeness requirement? This question also relates the debate on deflationism more deeply and broadly to the philosophy of logic and mathematics, since it involves the following questions: What axioms are needed to fully capture the relevant content of a given subject matter? How should truth be formally implemented in each context? In which case is the axiomatic approach with the assumption of (Syn) appropriately applied and essentially needed? What theory is 'rich' enough to make our mathematical commitment concerning the syntactic structure of formal languages negligibly insubstantial? What theories are maximally rich? And so on. There are more factors to be taken into account for the conservativeness argument to go through than philosophers seem to have previously thought, and we haven't yet reached the stage in which we can make a final verdict on the validity of the conservativeness argument: deflationary theories of truth over 'rich' subject matters have not yet been sufficiently explored, and more philosophical and mathematical developments in this area are to be awaited.

Let me finally emphasise again that most philosophical debates on the axiomatic theories of truth have so far been based on formal results about those theories over arithmetic, and this is presumably because philosophers believe that those formal results over arithmetic and their arguments on the basis of them can be generalised to other cases. However, this extrapolation is not valid as I have argued. More generally, theories of truth over arithmetic are often compared and related to second-order arithmetic, but recent research reveals that there are also a number of significant dissimilarities between second-order arithmetic and second-order theories over other bases such as set theory and the theory of real numbers; see Sato (2014, 2015); Schweber (2015) and Hachtman (2017). These formal results indicate that the arithmetical basis shows singular behaviour different from other bases. A primary source of the peculiarity of arithmetic is the $\Pi_{1}^{1}$-completeness of the notion of well-foundedness, and this fact is deeply related to the specific inductive nature of natural numbers in the sense that

39 Actually, I presented one more such type of the conservativeness argument in $\mathrm{fn} 20$ from the perspective of the logic-linguistic function of truth. 
$\mathbb{N}(=\omega)$ is the least infinite ordinal and only contains finite entities, which also plays the crucial role in the proof of Cieśliński's theorem. All these formal results and considerations seem to support my proposal.

Open Access This article is distributed under the terms of the Creative Commons Attribution 4.0 International License (http://creativecommons.org/licenses/by/4.0/), which permits unrestricted use, distribution, and reproduction in any medium, provided you give appropriate credit to the original author(s) and the source, provide a link to the Creative Commons license, and indicate if changes were made.

\section{References}

Achourioti, T., Galinon, H., Martínez-Fernández, J., \& Fujimoto, K. (Eds.). (2015). Unifying the philosophy of truth, volume 36 of logic, epistemology, and the unity of science. Heidelberg: Springer.

Aczel, P. (1980). Frege structures and the notion of proposition, truth and set. In J. Barwise, H. Keisler, \&

K. Kunen (Eds.), The kleene symposium (pp. 31-59). Amsterdam: North-Holland.

Azzouni, J. (1999). Comments on shapiro. Journal of Philosophy, 96, 541-544.

Beeson, M. (1985). Foundations of constructive mathematics. Berlin: Springer.

Cieśliński, C. (2010). Truth, conservativeness, and provability. Mind, 119, 409-422.

Corcoran, J., Frank, W., \& Maloney, M. (1974). String theory. The Journal of Symbolic Logic, 39, $625-637$.

Enayat, A., \& Visser, A. (2015). New constructions of satisfaction classes. In T. Achourioti, H. Galinon, J. Martínez-Fernández, \& K. Fujimoto (Eds.), Unifying the philosophy of truth, volume 36 of logic, epistemology, and the unity of science (pp. 321-335). Heidelberg: Springer.

Feferman, S. (1991). Reflecting on incompleteness. The Journal of Symbolic Logic, 56, 1-49.

Field, H. (1994). Deflationist views of meaning and content. Mind, 103, 247-285.

Field, H. (1999). Deflating the conservativeness argument. The Journal of Philosophy, 96, 533-540.

Fujimoto, K. (2012). Classes and truths in set theory. Annals of Pure and Applied Logic, 163, 1484-1523. Fujimoto, K. (2017). Compositional truths can catch up with non-compositional Truths (submitted).

Ganea, M. (2009). Arithmetic on semigroups. The Journal of Symbolic Logic, 74, 265-278.

Hachtman, S. (2017). Determinacy in third order arithmetic. Ann Pure Appl Logic. doi:10.1016/j.apal. 2017.05.004.

Hájek, P., \& Pudlak, P. (1993). Metamathematics of first-order arithmetic. Berlin: Springer.

Halbach, V. (1999). Disquotationalism and infinite conjunctions. Mind, 108, 1-22.

Halbach, V. (2001). How innocent is deflationism? Synthese, 126, 167-194.

Halbach, V. (2009). Reducing compositional to disquotational truth. The Review of Symbolic Logic, 2, $786-798$.

Halbach, V. (2010). Axiomatic theories of truth. Cambridge: Cambridge University Press.

Heck, R. (2009). The strength of truth theories. (unpublished manusctipt).

Horsten, L. (1995). The semantical paradoxes, the neutrality of truth, and the neutrality of the minimalist theory of truth. In P. Cortois (Ed.), The many problems of realism (pp. 173-187). Oxford: Tilburg University Press.

Horsten, L. (2011). The Tarskian turn. Cambridge, Massachusetts: The MIT Press.

Isaacson, D (1987). Arithmetical truth and hidden higher-order concepts. In The Paris Logic Group (Eds.),Logic Colloquium '85: proceedings of the colloquium held in Orsay, France July 1985, (pp. 147-169). Amsterdam: North Holland.

Ketland, J. (1999). Deflationism and tarski's paradise. Mind, 108, 69-94.

Ketland, J. (2005). Deflationism and gödel phenomena: Reply to tenannt. Mind, 114, 75-88.

Ketland, J. (2010). Truth, conservativeness, and provability: Reply to Cieśliński. Mind, 119, 423-436.

Leigh, G., \& Nicolai, C. (2013). Axiomatic truth, syntax and metatheoretic reasoning. Review of Symbolic Logic, 6, 613-636.

Montagna, F., \& Mancini, A. (1994). A minimal predicative set theory. Notre Dame Journal of Formal Logic, 35, 186-203.

Nicolai, C. (2015). Deflationary truth and the ontology of expressions. Synthese, 192, 4031-4055.

Rathjen, M. (1994). Proof theory of reflection. Annals of Pure and Applied Logic, 68, 181-224. 
Sato, K. (2014). Relative predicativity and dependent recursion in second-order set theory and higher-order theories. The Journal of Symbolic Logic, 79, 712-732.

Sato, K. (2015). Full and hat inductive definitions are equivalent in NBG. Archive for Mathematical Logic, 54, 75-112.

Schweber, N. (2015). Transfinite recursion in higher reverse mathematics. The Journal of Symbolic Logic, 80, 940-969.

Shapiro, S. (1998). Proof and truth: Through thick and thin. The Journal of Philosophy, 95, 493-521.

Shapiro, S. (2004). Deflation and conservation. In V. Halbach \& L. Horsten (Eds.), Principles of truth (2nd ed., pp. 103-128). Frankfurt: Ontos Verlag.

Sieg, W. (1999). Hilbert's programs: 1917-1922. The Bulletin of Symbolic Logic, 5, 1-44.

Simpson, S. G. (2009). Subsystems of second order arithmetic. Cambridge: Cambridge University Press.

Švejdar, V. (2009). On interpretability in the theory of concatenation. Notre Dame Journal of Formal Logic, 50, 87-95.

Tennant, N. (2002). Deflationism and the Gödel's phenomena. Mind, 111, 551-582.

Vänäänen, J. (2001). Second-order logic and foundation of mathematics. The Bulletin of Symbolic Logic, 7, 504-520. 